\title{
MHD mode coupling in the neighbourhood of a 2D null point ${ }^{\star}$
}

\author{
J. A. McLaughlin ${ }^{\star}$ and A. W. Hood
}

School of Mathematics and Statistics, University of St Andrews, KY16 9SS, UK

e-mail: james@mcs.st-and.ac.uk

Received 5 May 2006 / Accepted 4 August 2006

\section{ABSTRACT}

\begin{abstract}
Context. At this time there does not exist a robust set of rules connecting low and high $\beta$ waves across the $\beta \approx 1$ layer. The work here contributes specifically to what happens when a low $\beta$ fast wave crosses the $\beta \approx 1$ layer and transforms into high $\beta$ fast and slow waves.

Aims. The nature of fast and slow magnetoacoustic waves is investigated in a finite $\beta$ plasma in the neighbourhood of a twodimensional null point.

Methods. The linearised equations are solved in both polar and cartesian forms with a two-step Lax-Wendroff numerical scheme. Analytical work (e.g. small $\beta$ expansion and WKB approximation) also complement the work.

Results. It is found that when a finite gas pressure is included in magnetic equilibrium containing an $X$-type null point, a fast wave is attracted towards the null by a refraction effect and that a slow wave is generated as the wave crosses the $\beta \approx 1$ layer. Current accumulation occurs close to the null and along nearby separatrices. The fast wave can now pass through the origin due to the non-zero sound speed, an effect not previously seen in related papers but clear seen for larger values of $\beta$. Some of the energy can now leave the region of the null point and there is again generation of a slow wave component (we find that the fraction of the incident wave converted to a slow wave is proportional to $\beta$ ). We conclude that there are two competing phenomena; the refraction effect (due to the variable Alfvén speed) and the contribution from the non-zero sound speed.

Conclusions. These experiments illustrate the importance of the magnetic topology and of the location of the $\beta \approx 1$ layer in the system.
\end{abstract}

Key words. magnetohydrodynamics (MHD) - waves - Sun: corona - Sun: magnetic fields - Sun: oscillations

\section{Introduction}

MHD wave motions have recently been observed in the Sun's atmosphere with the SOHO and TRACE satellites, see for example the detection of slow magnetoacoustic waves by Berghmans \& Clette (1999) and De Moortel et al. (2000) and of fast magnetoacoustic waves by Nakariakov et al. (1999). It is clear that the local coronal magnetic field plays a key role in determining their propagation properties.

To begin to understand this inhomogeneous magnetised environment, it is useful to look at the structure (topology) of the magnetic field itself. Potential field extrapolations of the coronal magnetic field can be made from photospheric magnetograms. Such extrapolations show the existence of an important feature of the topology; null points. Null points are points in the field where the Alfvén speed is zero. Detailed investigations of the coronal magnetic field, using such potential field calculations, can be found in Beveridge et al. (2002) and Brown \& Priest (2001).

Building on the earlier work of Bulanov \& Syrovatskii (1980) and Craig \& Watson (1992), McLaughlin \& Hood (2004) found that for a single $2 \mathrm{D}$ null point, the fast magnetoacoustic wave was attracted to the null and the wave energy accumulated there. In addition, they found that the Alfvén wave energy accumulated along the separatrices, the topological feature that

$\star$ Appendices A, B are only available in electronic form at http: //wwW . aanda. org

$\star \star$ Present address: The Catholic University of America, NASA Goddard Space Flight Center, Code 612.1, Greenbelt, MD 20771, USA. separates regions with different magnetic flux connectivity. Their paper looked at MHD wave propagation in a $\beta=0$ plasma. The aim of this paper is to extend their model to include plasma pressure (finite $\beta$ plasma). The most obvious effect is the introduction of slow magnetoacoustic waves. The fast wave can now also pass through the null point (as we have a non-zero fast wave speed there due to the finite sound speed) and, thus, perhaps carry wave energy away from that area. There could also be coupling and wave conversion near the location where the sound speed and Alfvén speed become comparable in magnitude. However, the exact nature of such coupling in MHD is unknown and will be looked at here. The behaviour of the Alfvén waves is unaffected by a finite $\beta$ (the plasma pressure plays no role in its propagation) and so the description by McLaughlin \& Hood (2004) remains valid in this linear, $2 \mathrm{D}$ regime.

Waves in the neighbourhood of a single $2 \mathrm{D}$ null point have been investigated by various authors. Bulanov \& Syrovatskii (1980) provided a detailed discussion of the propagation of fast and Alfvén waves using cylindrical symmetry. Craig \& Watson (1992) mainly consider the radial propagation of the $m=0$ mode (where $m$ is the azimuthal wavenumber) using a mixture of analytical and numerical solutions. They show that the propagation of the $m=0$ wave towards the null point generates an exponentially large increase in the current density and that magnetic resistivity dissipates this current in a time related to $\log \eta$. Craig \& McClymont $(1991,1993)$ investigate the normal mode solutions for both $m=0$ and $m \neq 0$ modes with resistivity included. Again, they emphasise that the current builds up as the inverse square of the radial distance from the null point. All these 
investigations were carried out using cylindrical models in which the generated waves encircled the null point. In a sense, there is nowhere else for the wave to propagate except into the null point.

A very detailed and comprehensive set of 2D numerical simulations of wave propagation in a stratified magneto-atmosphere was conducted by Rosenthal et al. (2002) and Bogdan et al. (2003). In these simulations, an oscillating piston generated both fast and slow MHD waves on a lower boundary and sent these waves up into the stratified, magnetised plasma. Their calculations showed that there was coupling between the fast and slow waves, and that this coupling was confined to a thin layer where the sound speed and the Alfvén velocity are comparable in magnitude, i.e. where the plasma-beta approaches unity. Away from this conversion zone, the waves were decoupled as either the magnetic pressure or plasma pressure dominated. In this, their papers and ours have a similar goal; to see how the topology affects the propagation of MHD waves, in a 2D system where the ratio of the sound speed to the Alfvén speed varys along every magnetic line of force.

Other authors have also looked at MHD mode coupling. Cally \& Bogdan (1997) describes 2D simulations in which both $f$-modes and $p$-modes are (partially) converted to slow magnetoacoustic gravity waves, due to strong gravitational stratification. De Moortel et al. (2004) investigated driving slow waves on the boundary of a 2D geometry with a horizontal density variation. They found coupling between slow and fast waves and phase mixing of the slow waves. The coupling of different wave modes has also been investigated by Ferraro \& Plumpton (1958), Zhugzhd \& Dzhalilov (1982) (with Meijer G-functions) and Cally (2001) (with hypergeometric ${ }_{2} F_{3}$ functions). All these works considered mode coupling through a gravitational stratification (vertical density inhomogenity) and, in particular, investigated the propagation of waves from a high $\beta$ to a low $\beta$ plasma. As waves propagate towards a null point, it is the nature of low $\beta$ to high $\beta$ propagation that is important. Finally, the coupling of fast waves and Alfvén waves has been investigated by Parker (1991) (linear with a density gradient) and by Nakariakov et al. (1997) (nonlinear excitation).

The paper has the following outline. In Sect. 2, the basic equations are described and the importance of the plasma $\beta$ discussed. The results for the fast and slow magnetoacoustic waves are presented for a reference value of the plasma $\beta$, namely $\beta_{0}=0.25$ in Sect. 3. Section 4 provides an interpretation of our results, with a discussion of mode conversion in Sect. 5. The robustness of our results and a clearer demonstration of mode conversion is shown in Sect. 6 for $\beta_{0}=2.25$. The conclusions are given in Sect. 7 and the appendices provide analytical approximations.

\section{Basic equations}

The usual MHD equations appropriate to the solar corona are used, with pressure and resistivity included. Hence,

$$
\begin{aligned}
\rho\left[\frac{\partial \boldsymbol{v}}{\partial t}+(\boldsymbol{v} \cdot \nabla) \boldsymbol{v}\right] & =\frac{1}{\mu}(\nabla \times \boldsymbol{B}) \times \boldsymbol{B}-\nabla p, \\
\frac{\partial \boldsymbol{B}}{\partial t} & =\nabla \times(\boldsymbol{v} \times \boldsymbol{B})+\eta \nabla^{2} \boldsymbol{B}, \\
\frac{\partial \rho}{\partial t}+\nabla \cdot(\rho \boldsymbol{v}) & =0 \\
\frac{\partial p}{\partial t}+(\boldsymbol{v} \cdot \nabla) p & =-\gamma p \nabla \cdot \boldsymbol{v}
\end{aligned}
$$

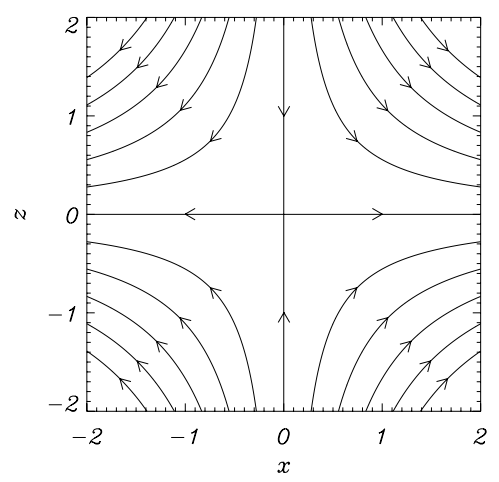

Fig. 1. The equilibrium equilibrium magnetic field and coordinate system.

where $\rho$ is the mass density, $\boldsymbol{v}$ is the plasma velocity, $\boldsymbol{B}$ the magnetic induction (usually called the magnetic field), $p$ is the plasma pressure, $\mu=4 \pi \times 10^{-7} \mathrm{Hm}^{-1}$ the magnetic permeability, $\eta=1 / \mu \sigma$ is the magnetic diffusivity $\left(\mathrm{m}^{2} \mathrm{~s}^{-1}\right)$ and $\sigma$ the electrical conductivity.

\subsection{Basic equilibrium}

The basic potential magnetic field structure is taken as a simple two dimensional, X-type neutral point. There are a lot of complicated effects including mode conversion and coupling; and a 2D geometry allows one to understand these effects better, before the extension to 3D. Therefore, the equilibrium magnetic field (see Fig. 1) is taken as

$\boldsymbol{B}_{0}=B\left(\frac{x}{a}, 0,-\frac{z}{a}\right)$

where $B$ is a characteristic field strength and $a$ is the length scale for magnetic field variations. Obviously, this configuration is no longer valid far from the null point, as the field strength tends to infinity. However, McLaughlin \& Hood (2006) looked at a magnetic field with a field strength that decays far from the null (again for a $\beta=0$ plasma) and they found that the key results from McLaughlin \& Hood (2004) remain valid close to the null.

As in McLaughlin \& Hood (2004), the linearised MHD equations are used to study the nature of the wave propagation near the null point. Using subscript 0 for equilibrium quantities and 1 for perturbed quantities, the linearised equation of motion becomes

$\rho_{0} \frac{\partial \boldsymbol{v}_{1}}{\partial t}=\left(\frac{\nabla \times \boldsymbol{B}_{1}}{\mu}\right) \times \boldsymbol{B}_{0}-\nabla p_{1}$,

the linearised induction equation

$\frac{\partial \boldsymbol{B}_{1}}{\partial t}=\nabla \times\left(\boldsymbol{v}_{1} \times \boldsymbol{B}_{0}\right)+\eta \nabla^{2} \boldsymbol{B}_{1}$,

the linearised equation of mass continuity

$\frac{\partial \rho_{1}}{\partial t}+\nabla \cdot\left(\rho_{0} \boldsymbol{v}_{1}\right)=0$

and the adiabatic energy equation

$\frac{\partial p_{1}}{\partial t}=-\gamma p_{0}\left(\nabla \cdot \boldsymbol{v}_{1}\right)$

We will not discuss Eq. (4) further as it can be solved once we know $\boldsymbol{v}_{1}$. We assume the background density and gas pressure 

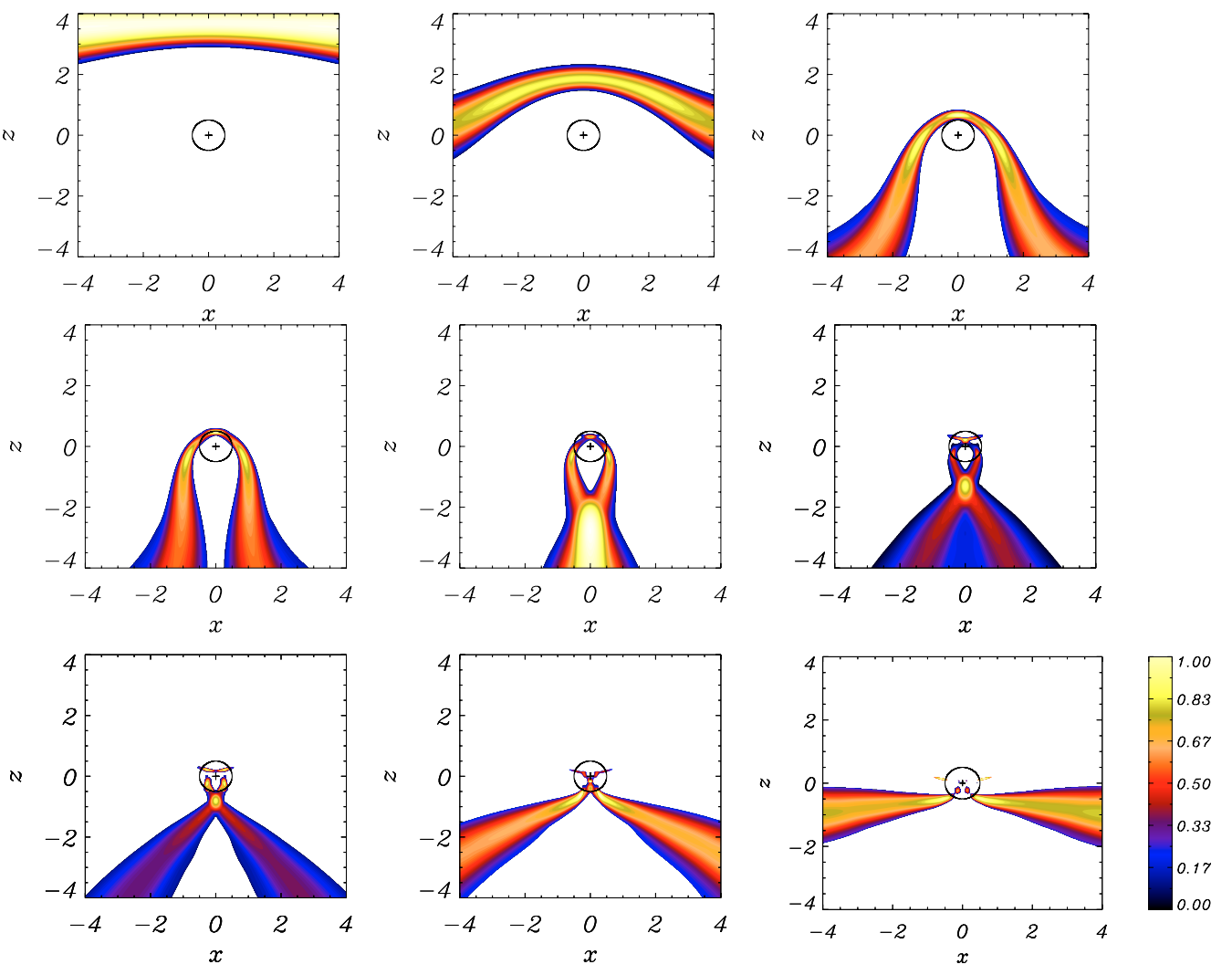

Fig. 2. Contours of $v_{\perp}$ for numerical simulation for a wave sent in from the upper boundary for $-4 \leq$ $x \leq 4$ and $\beta_{0}=0.25$ and its resultant propagation at times a) $t=0.33$; b) $t=1.0$; c) $t=2.0$; d) $t=2.33$; e) $t=2.67$; f) $t=$ 3.0 ; g) $t=3.33$; h) $t=3.67$ and i) $t=4.0$, labelling from top left to bottom right. The black circle indicates the position of the $c_{\mathrm{s}}=v_{\mathrm{A}}$ layer. The cross denotes the null point in the magnetic configuration. are uniform and labelled as $\rho_{0}$ and $p_{0}$ respectively. However, we note that a spatial variation in $\rho_{0}$ can cause phase mixing (Heyvaerts \& Priest 1983; De Moortel et al. 1999; Hood et al. 2002).

\subsection{Coordinate system and non-dimensionalisation}

The linearised velocity, $\boldsymbol{v}_{1}$, is split into components parallel and perpendicular to the equilibrium magnetic field. Thus,

$\boldsymbol{v}_{1}=V_{\|}\left(\frac{\boldsymbol{B}_{0}}{\sqrt{\boldsymbol{B}_{0} \cdot \boldsymbol{B}_{0}}}\right)-V_{\perp}\left(\frac{\nabla A_{0}}{\sqrt{\boldsymbol{B}_{0} \cdot \boldsymbol{B}_{0}}}\right)+v_{y} \hat{\boldsymbol{y}}$

where $A_{0}=-B x z / a$ is the $y$-component of the vector magnetic potential. The terms in brackets are unit vectors. Splitting the velocity into components parallel and perpendicular to the equilibrium magnetic field makes MHD mode detection and interpretation easier. For example, in a low $\beta$ plasma, the slow wave is guided by the magnetic field and has a velocity component that is mainly field-aligned. This makes perfect sense when $\beta \ll 1$ but its usefulness is less clear when $\beta \gg 1$ near the null point.

To aid the numerical calculation, our primary variables are considered to be $v_{\perp}=\sqrt{\boldsymbol{B}_{0} \cdot \boldsymbol{B}_{0}} V_{\perp}$ and $v_{\|}=\sqrt{\boldsymbol{B}_{0} \cdot \boldsymbol{B}_{0}} V_{\|}$.

Taking $v_{y}=0$ means we do not consider the Alfvén wave here as the description by McLaughlin \& Hood (2004) is still valid (the plasma pressure plays no role in its propagation).

We non-dimensionalise all variables by setting $\boldsymbol{v}_{1}=v_{0} \boldsymbol{v}_{1}^{*}$, $v_{\perp}=v_{0} B v_{\perp}^{*}, v_{\|}=v_{0} B v_{\|}^{*}, \boldsymbol{B}_{0}=B \boldsymbol{B}_{0}^{*}, \boldsymbol{B}_{1}=B \boldsymbol{B}_{1}^{*}, x=a x^{*}, z=a z^{*}$, $p_{1}=p_{0} p_{1}^{*}, \nabla=\frac{1}{a} \nabla^{*}, t=t_{0} t^{*}, A_{0}=a B A_{0}^{*}$ and $\eta=\eta_{0}$, where we let $*$ denote a dimensionless quantity and $v_{0}, B, a, p_{0}, t_{0}$ and $\eta_{0}$ are constants with the dimensions of the variable they are scaling. We then set $\frac{B}{\sqrt{\mu \rho_{0}}}=v_{0}$ and $v_{0}=\frac{a}{t_{0}}$ (i.e. we measure our speed in units of $v_{0}$, which can be thought of as a constant background Alfvén speed). We also set $\frac{\eta_{0} t_{0}}{a^{2}}=R_{\mathrm{m}}^{-1}$, where $R_{\mathrm{m}}$ is the magnetic
Reynolds number, and set $\beta_{0}=\frac{2 \mu p_{0}}{B^{2}}$, where $\beta_{0}$ is the plasma $\beta$ at a distance unity from the origin (see Sect. 2.4). Thus, we generate the non-dimensionalised versions of Eqs. (2), (3) and (5) and under these scalings, $t^{*}=1$ (for example) refers to $t=t_{0}=\frac{a}{v_{0}}$; i.e. the time taken to travel a distance $a$ at the reference background Alfvén speed. For the rest of this paper, we drop the star indices; the fact that they are now non-dimensionalised is understood.

\subsection{Linearised equations}

The linearised equations are:

$$
\begin{aligned}
\frac{\partial v_{\perp}}{\partial t}= & v_{\mathrm{A}}^{2}(x, z)\left(\frac{\partial b_{z}}{\partial x}-\frac{\partial b_{x}}{\partial z}\right)-\frac{\beta_{0}}{2}\left(z \frac{\partial p_{1}}{\partial x}+x \frac{\partial p_{1}}{\partial z}\right) \\
\frac{\partial v_{\|}}{\partial t}= & -\frac{\beta_{0}}{2}\left(x \frac{\partial p_{1}}{\partial x}-z \frac{\partial p_{1}}{\partial z}\right) \\
\frac{\partial b_{x}}{\partial t}= & -\frac{\partial v_{\perp}}{\partial z}+\frac{1}{R_{\mathrm{m}}}\left(\frac{\partial^{2} b_{x}}{\partial x^{2}}+\frac{\partial^{2} b_{x}}{\partial z^{2}}\right) \\
\frac{\partial b_{z}}{\partial t}= & \frac{\partial v_{\perp}}{\partial x}+\frac{1}{R_{\mathrm{m}}}\left(\frac{\partial^{2} b_{z}}{\partial x^{2}}+\frac{\partial^{2} b_{z}}{\partial z^{2}}\right) \\
\frac{\partial p_{1}}{\partial t}= & \frac{-\gamma}{x^{2}+z^{2}}\left[\left(x \frac{\partial v_{\|}}{\partial x}-z \frac{\partial v_{\|}}{\partial z}\right)-2 \frac{x^{2}-z^{2}}{x^{2}+z^{2}} v_{\|}\right. \\
& \left.+\left(z \frac{\partial v_{\perp}}{\partial x}+x \frac{\partial v_{\perp}}{\partial z}\right)-\frac{4 x z}{x^{2}+z^{2}} v_{\perp}\right]
\end{aligned}
$$

where the dimensionless Alfvén speed, $v_{\mathrm{A}}(x, z)=\sqrt{x^{2}+z^{2}}$. Note that by taking $\beta_{0}=0$ and ignoring resistivity, $\left(R_{\mathrm{m}} \rightarrow \infty\right)$, we recover the equations discussed in McLaughlin \& Hood (2004). 

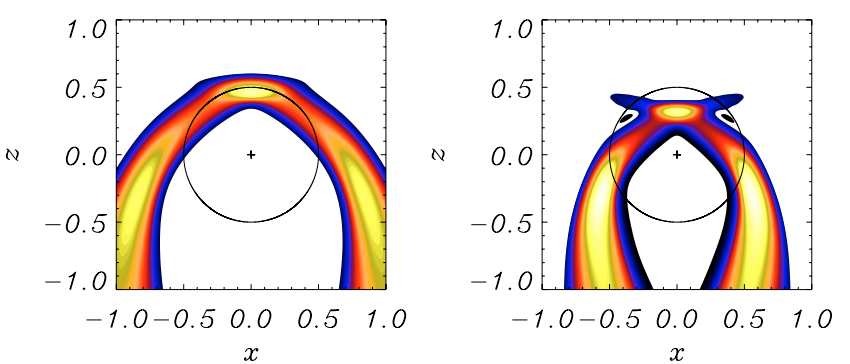

\subsection{Plasma $\beta$}

The parameter of key importance in Eqs. (6) is $\beta_{0}$. The plasma $\beta$ is defined as the ratio of the thermal plasma pressure to the magnetic pressure. In most parts of the corona, the plasma $\beta$ is much less than unity but, near null points, the magnetic field strength is small (and is zero at the null) and the plasma $\beta$ becomes large. There is also coupling between the perpendicular and parallel velocity components when $\beta_{0} \neq=0$ and this coupling is most effective where the sound speed and the Alfvén velocity are comparable in magnitude. Bogdan et al. (2003) call this zone the magnetic canopy or the $\beta \approx 1$ layer.

The plasma $\beta$ varies throughout the whole region and

$\beta=\frac{2 \mu p_{0}}{B^{2}} \frac{1}{x^{2}+z^{2}} \Rightarrow \beta=\frac{\beta_{0}}{x^{2}+z^{2}}=\frac{\beta_{0}}{r^{2}}$,

where $r^{2}=x^{2}+z^{2}$. Therefore, the $\beta=1$ layer occurs at a radius $r=\sqrt{\beta_{0}}$, where the gas pressure is equal to the magnetic pressure. However, in a uniform plasma, it is not the $\beta=1$ layer that is most important, but instead it is where the sound speed is equal to the Alfvén speed, i.e. $c_{\mathrm{s}}=v_{\mathrm{A}}$. Recalling that $c_{\mathrm{s}}=\sqrt{\frac{\gamma p_{0}}{\rho_{0}}}=\sqrt{\frac{\gamma \beta_{0}}{2} \frac{B^{2}}{\mu \rho_{0}}}$ (which implies $c_{\mathrm{s}}^{*}=\sqrt{\frac{\gamma}{2} \beta_{0}}$ in nondimensional units), we see that for the present non-uniform equilibrium $c_{\mathrm{s}}=v_{\mathrm{A}}$ at a radius $r=\sqrt{\frac{\gamma}{2} \beta_{0}}$ and it is when the incoming wave passes through this layer that the mode coupling occurs. Of course, the difference between the $\beta=1$ layer at $r=\sqrt{\beta_{0}}$ and the $c_{\mathrm{s}}=v_{\mathrm{A}}$ layer at $r=\sqrt{\frac{\gamma}{2} \beta_{0}}$ is very small, and, hence, it is easier to refer to this as the $\beta \approx 1$ layer.

Finally, we note that the basic fast and slow wave speeds for this equilibrium are given in dimensionless form as

$$
c_{\text {fast }}^{2}=\frac{\gamma}{2} \beta_{0}+r^{2}, \quad c_{\text {slow }}^{2}=\frac{r^{2} \gamma \beta_{0} / 2}{\gamma \beta_{0} / 2+r^{2}} .
$$

\section{MHD wave propagation with $\beta_{0}=0.25$}

In this section, the linearised MHD equations, namely Eqs. (6), are solved numerically using a two-step Lax-Wendroff scheme. In dimensionless units, the numerical domain is $-4 \leq x \leq 4$ and $-4 \leq z \leq 4$ and initially we consider a single wave pulse coming in from the top boundary due to a disturbance in the perpendicular component of the velocity. For the single wave pulse, the boundary conditions are chosen such that:

$$
\begin{aligned}
& v_{\perp}(x, 4)=\left\{\begin{array}{c}
\sin \omega t \text { for } 0 \leq t \leq \frac{\pi}{\omega}, \quad v_{\|}(x, 4)=0, \\
0 \quad \text { otherwise }
\end{array}\right. \\
& \left.\frac{\partial v_{\perp}}{\partial x}\right|_{x=-4}=0,\left.\quad \frac{\partial v_{\perp}}{\partial x}\right|_{x=4}=0,\left.\quad \frac{\partial v_{\perp}}{\partial z}\right|_{z=-4}=0, \\
& \left.\frac{\partial v_{\|}}{\partial x}\right|_{x=-4}=0,\left.\quad \frac{\partial v_{\|}}{\partial x}\right|_{x=4}=0,\left.\quad \frac{\partial v_{\|}}{\partial z}\right|_{z=-4}=0 .
\end{aligned}
$$

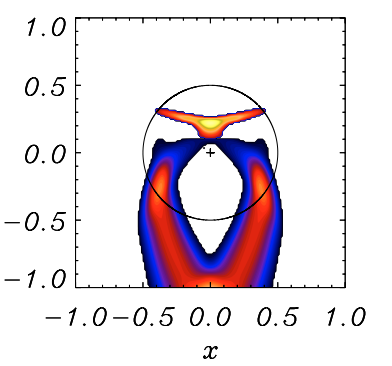

Fig. 3. Blow-up subfigures of $v_{\perp}$ from Fig. 2 at times a) $t=2.33$; b) $t=2.67$ and c) $t=3.0$, labelling left to right.

Tests show that the central behaviour is largely unaffected by the choices of side and bottom boundary conditions. The other boundary conditions on the perturbed magnetic field follow from the remaining equations and the solenoidal condition.

The location of the upper boundary is not important and it is the value of $\beta_{0}$ that determines the distance the fast wave travels before encountering the mode conversion region. Initially, we set $\beta_{0}=0.25$ and $R_{\mathrm{m}}=10^{3}$. We also take $\omega=2 \pi$, as in McLaughlin $\& \operatorname{Hood}(2004)$. The results for $v_{\perp}$ can be seen in Figs. 2 and 3 and for $v_{\|}$in Fig. 4.

\subsection{Initial disturbance in the perpendicular component}

In the zero $\beta$ limit (see McLaughlin \& Hood 2004), the wave pulse given above is a fast MHD disturbance, but for the present problem this is no longer true. In the system presented here, the fast wave is no longer purely described by a perpendicular component: it is predominately given by the perpendicular component but also has a smaller parallel component. Thus, the single wave pulse described above is a disturbance in the perpendicular component, as opposed to simply a fast MHD disturbance. This difference in definition will be important when we come to interpret the different wave types in Sect. 4. In addition, it can be seen that the governing Eqs. (6) that $v_{\perp}$ acts as a driver for $v_{\|}$. Since the value of the plasma $\beta$ is small on the upper boundary, $v_{\perp}$ initially behaves in the same manner as the zero $\beta$ case. However, the parallel component of the velocity is now driven by the perpendicular component.

Thus, the general solution for $v_{\|}$will consist of two parts; a complementary function, corresponding to a slow mode disturbance and a particular integral, due to the fast mode driver term. These two parts to the $v_{\|}$wave are clearly seen in the simulations. However, it is clearer if we first discuss the behaviour of the $v_{\perp}$ component before moving on to the parallel component.

\subsection{Behaviour of $v_{\perp}$}

We find that the $v_{\perp}$ disturbance travels towards the neighbourhood of the null point and begins to wrap around it. This is due to refraction caused by the spatially varying Alfvén speed, $v_{\mathrm{A}}(x, z)$, as noted by Nakariakov \& Roberts (1995) and seen in McLaughlin \& Hood (2004) (further references are cited in the latter). Once the wave reaches the $c_{\mathrm{s}}=v_{\mathrm{A}}$ layer (denoted by the black circle around the null in Fig. 2) the nature of the wave changes; part of the wave now appears to spread out along the field lines (this part also moves slower than the rest of the wave). Meanwhile, the majority of the wave outside the circle continues to refract about the null. This wrapping effect repeats, and each time part of the wave crosses the $c_{\mathrm{s}}=v_{\mathrm{A}}$ layer, part of it is transformed to the field-guided wave. Figure 3 shows a blowup of subfigures $(d)$ to $(f)$ during the time of the first crossing. In this figure, we can clearly see the splitting of the wave as it nears and then crosses the $c_{\mathrm{s}}=v_{\mathrm{A}}$ layer. Thus, we can see that 

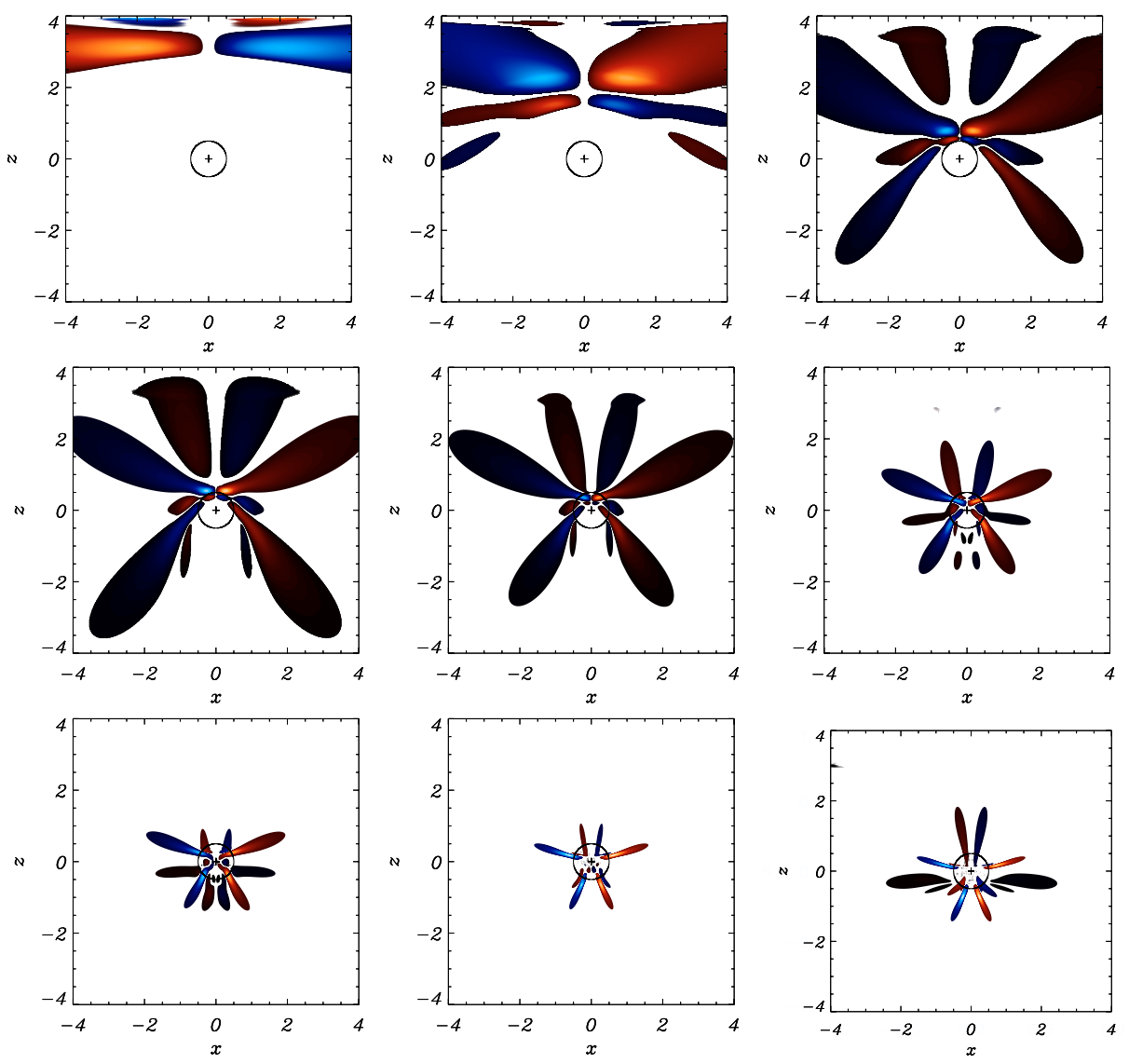

Fig. 4. Contours of $v_{\|}$for numerical simulation for a fast wave sent in from upper boundary for $-4 \leq x \leq 4$ and $\beta_{0}=$ 0.25 and its resultant propagation at times a) $t=0.33$; b) $t=$ 1.0 ; c) $t=2.0$; d) $t=2.33$; $t=$ 2.67 ; f) $t=3.0$; g) $t=3.33$; h) $t=3.67$ and i) $t=4.0$, labelling from top left to bottom right. The black circle indicates the position of the $c_{\mathrm{s}}=v_{\mathrm{A}}$ layer. The cross denotes the null point in the magnetic configuration. there are three main types of wave behaviour in the perpendicular component; outside the $c_{\mathrm{s}}=v_{\mathrm{A}}$ layer we predominatrely see the refraction effect, whereas inside we see both fast and slow wave behaviour; the latter which appears as a field-guided wave. We shall give a fuller description of this phenomenon in Sect. 6 and give an interpretation of these different wave types in Sect. 4.

\subsection{Behaviour of $v_{\|}$}

The behaviour of $v_{\|}$can be seen in Fig. 4. We see that it is an antisymmetric wave about the $z$ axis, and that it is focusing in towards the null. This pulse travels at the same speed as the perpendicular component wave, namely the fast wave speed. This is not surprising since, as mentioned previously, $v_{\perp}$ drives $v_{\|}$. Thus, mathematically this leading wave can be thought of as the particular solution to an (inhomogeneous) equation. Note that the range of the colour scale indicates that the maximum amplitude of $v_{\|}$is 0.2 and this is approximately the same size as $\beta_{0}$. We use this relatively weak coupling to construct an approximate analytic solution (see Appendix A). There is also a complementary function part that satisfies the homogeneous equation

$\frac{\partial^{2} v_{\|_{\mathrm{CF}}}}{\partial t^{2}}=\frac{\gamma \beta_{0}}{2}\left(\boldsymbol{B}_{0} \cdot \nabla\right)^{2}\left(\frac{v_{\|_{\mathrm{CF}}}}{B_{0}^{2}}\right)$.

This has a solution that involves a function of the variable

$t+\sqrt{\frac{2}{\gamma \beta_{0}}} \log z$.

The complementary function part can be seen in Fig. 5. This second part of the solution lags behind the particular solution and propagates at the slow wave speed. In a low $\beta$ plasma this is approximately the sound speed and hence is proportional to $\sqrt{\beta_{0}}$. From the lower subfigures of Fig. 5, we see that the complementary function does indeed travel with a speed proportional to $\sqrt{\beta_{0}}$, and has an amplitude that varies as $\beta_{0}^{1.6}$. The complementary function has a discontinuous shape (as is also shown in Appendix A) and its amplitude is small, $O\left(\beta_{0}^{1.6}\right)$, compared to the particular integral, $O\left(\beta_{0}\right)$, and is thus difficult to see in Fig. 4.

There are also several other noteworthy aspects to Fig. 4. Firstly, it is obviously more complicated than the $v_{\perp}$ wave. Secondly, this component has eight lobe-like structures by the time $t=2.0$, when the leading edge of the wave is about to cross the region where $c_{\mathrm{S}}=v_{\mathrm{A}}$. The lobes alternate between positive and negative values. This complicated lobe-like structures can be explained by considering the same system but driven by a circular boundary condition. This is explained in Appendix A, where the main conclusions is that the equilibrium magnetic configuration naturally leads to a $\sin 4 \theta$ dependence in $v_{\|}$. This explains the complicated, lobe-like structuring, i.e. the lobes come from the equilibrium magnetic configuration (but the wave is no longer circular so the $\sin 4 \theta$ is less apparent).

\section{Interpretation}

Roberts (1985) showed that $\boldsymbol{v}_{\text {Alfvén }}, \boldsymbol{v}_{\text {slow }}$ and $\boldsymbol{v}_{\text {fast }}$ form an orthogonal basis of vectors for the linearised MHD equations in a uniform plasma. In this paper, we do not consider the Alfvén wave and so our 2D system can be described in terms of the vectors $\boldsymbol{v}_{\text {fast }}$ and $\boldsymbol{v}_{\text {slow }}$. Due to the form of the equilibrium magnetic field near the null point, we choose to work in the directions parallel and perpendicular to the magnetic field and thus we may 

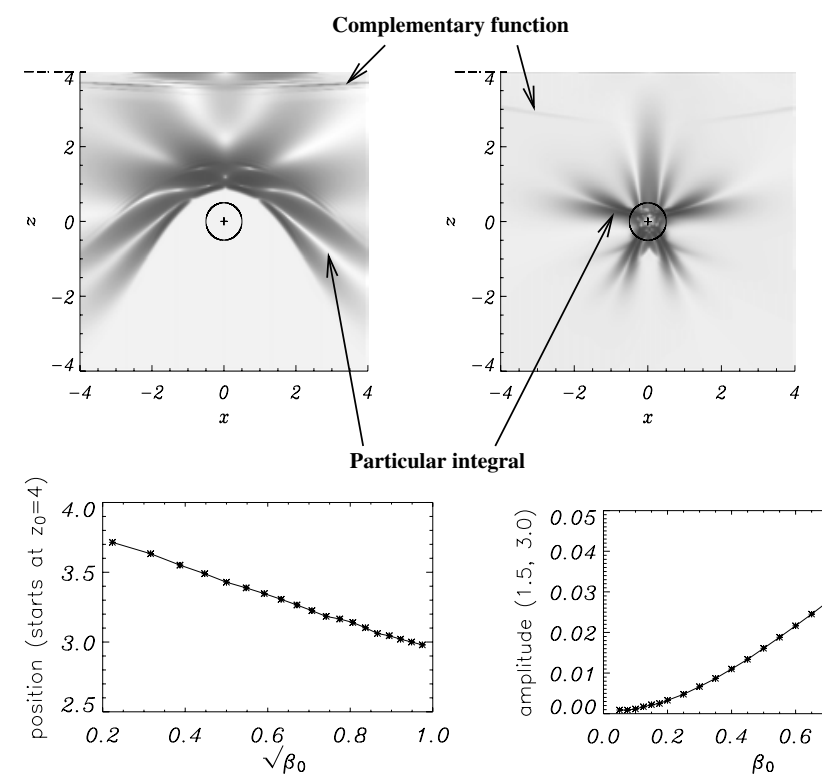

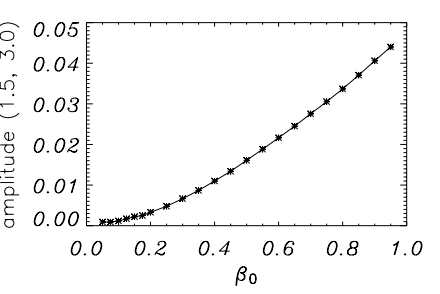

Fig. 5. Shaded surface of $v_{\|}$for numerical simulation for a fast wave (pulse) sent in from upper boundary for $-4 \leq x \leq 4$ and $\beta_{0}=0.25$ at time $t=1.5$ (top left) and $t=3.5$ (top right). The bottom left figure shows the position reached by the complementary function along $x=1.5$ after $t=1.5$ (the gradient of the straight line based on the first and last points is -0.98 . The bottom right figure shows the amplitude of the complementary function as a function of $\beta_{0}$. The amplitude is proportional to $\beta_{0}^{1.6}$, although $\beta_{0}^{1.5}$ is to be expected from the boundary conditions.

Table 1. Properties of fast and slow waves depending on their environment.

\begin{tabular}{|c|c|c|}
\hline & Fast wave & Slow wave \\
\hline High $\beta$ & $\begin{array}{l}\text { Behaves like isotropic sound wave } \\
\left.\text { (speed } c_{\mathrm{s}}\right)\end{array}$ & $\begin{array}{c}\text { Guided along } \boldsymbol{B}_{0} \\
\text { Transverse wave propagating at speed } v_{\mathrm{A}} \\
\text { Dominant velocity component is } v_{\perp}\end{array}$ \\
\hline Low $\beta$ & $\begin{array}{l}\text { Propagates roughly isotropically } \\
\text { (speed } v_{\mathrm{A}} \text { ) } \\
\text { Dominant velocity component is } v_{\perp}\end{array}$ & $\begin{array}{c}\text { Guided along } \boldsymbol{B}_{0} \\
\text { Longitudinal wave propagating at speed } c_{T} \\
\text { Dominant velocity component is } v_{\|}\end{array}$ \\
\hline
\end{tabular}

represent these two vectors in terms of $\boldsymbol{v}_{\text {fast }}$ and $\boldsymbol{v}_{\text {slow }}$, i.e.

$\boldsymbol{v}_{\perp}=\mathcal{A} \boldsymbol{v}_{\text {fast }}+\mathcal{B} \boldsymbol{v}_{\text {slow }}, \quad \boldsymbol{v}_{\|}=C \boldsymbol{v}_{\text {fast }}+\mathcal{D} \boldsymbol{v}_{\text {slow }}$

where $\mathcal{A}, \mathcal{B}, C$ and $\mathcal{D}$ are unknown functions that depend upon the magnetic geometry (and possibly the plasma $\beta$ ). Equivalently, we may represent $\boldsymbol{v}_{\text {fast }}$ and $\boldsymbol{v}_{\text {slow }}$ in terms of $\boldsymbol{v}_{\perp}$ and $\boldsymbol{v}_{\|}$. This representation is only possible because both $\boldsymbol{v}_{\text {fast }}$ $\& \boldsymbol{v}_{\text {slow }}$ and $\boldsymbol{v}_{\perp} \& \boldsymbol{v}_{\|}$are linearly independent vectors. Note that in a low $\beta$ region or when the plasma is nearly uniform, $\mathcal{B}$ will be very small. Thus in these regions, we interpret $\boldsymbol{v}_{\perp}$ as predominately a fast wave (i.e. $\boldsymbol{v}_{\perp} \approx \mathcal{A}\left(\boldsymbol{v}_{\text {fast }}\right)$.

However, we must be careful. The concepts of fast and slow waves were derived for a unidirectional magnetic field (see Edwin \& Roberts 1983) and so these ideas may not carry over to more complicated geometries quite as simply as claimed here. Nonetheless, we shall continue to use terms such as fast and slow wave in our interpretation of the waves in this paper (i.e. we shall use the terminology and intuition gained from the unidirectional magnetic field model). Thus, we choose to interpret the waves seen in our perpendicular and parallel velocities using the terminology of fast and slow waves.

\section{Mode conversion across the $c_{\mathrm{s}}=v_{\mathrm{A}}$ layer}

The $c_{\mathrm{S}}=v_{\mathrm{A}}$ layer is of critical importance to our system. When the Alfvén speed and sound speed are dissimilar, there is negligible coupling between the fast and slow magnetoacoustic waves. However, near the $c_{\mathrm{s}}=v_{\mathrm{A}}$ layer, the two waves can resonantly interact with each other and strong mode coupling can occur. Since $\beta=\frac{\beta_{0}}{x^{2}+z^{2}}$ (where $\beta_{0}$ is a constant of our choosing), our system consists of a region of low $\beta$ plasma outside the $c_{\mathrm{s}}=v_{\mathrm{A}}$ layer and a region of high $\beta$ plasma within (see Fig. 6). Fast and slow

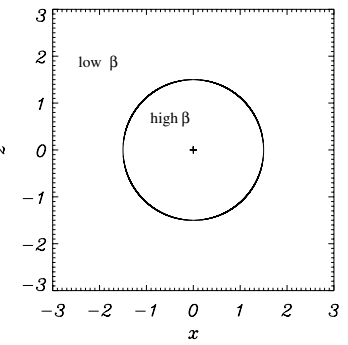

Fig. 6. Regions of high and low $\beta$ in our equilibrium magnetic field, where $\beta=\frac{\beta_{0}}{x^{2}+z^{2}}$. The black circle indicates the position of the $c_{\mathrm{s}}=$ $v_{\mathrm{A}}$ layer and the cross denotes the null point.

waves have differing properties depending on if they are in a high or low $\beta$ environment (see e.g. Bogdan et al. 2003). See Table 1 for a summary of these properties.

Thus, in a low $\beta$ region, the velocity vector of the fast wave is predominately perpendicular to the equilibrium magnetic field, although the wave can propagate almost isotropically. The slow wave has a velocity component along the field and propagates along $\boldsymbol{B}_{0}$. In the high $\beta$ region, the fast wave again propagates isotropically but this time the velocity vector is parallel to the wavenumber vector. Hence, the fast wave now has components of velocity that are perpendicular and parallel to the field. The slow wave is again guided by the field but the velocity is perpendicular to $\boldsymbol{B}_{0}{ }^{1}$.

\footnotetext{
1 Note that the high $\beta$ slow wave shows up in the perpendicular component because it is a transverse wave $(\boldsymbol{v} \cdot \boldsymbol{k}=0)$ and not because it is propagating across the field; the slow wave cannot propagate across the field!
} 

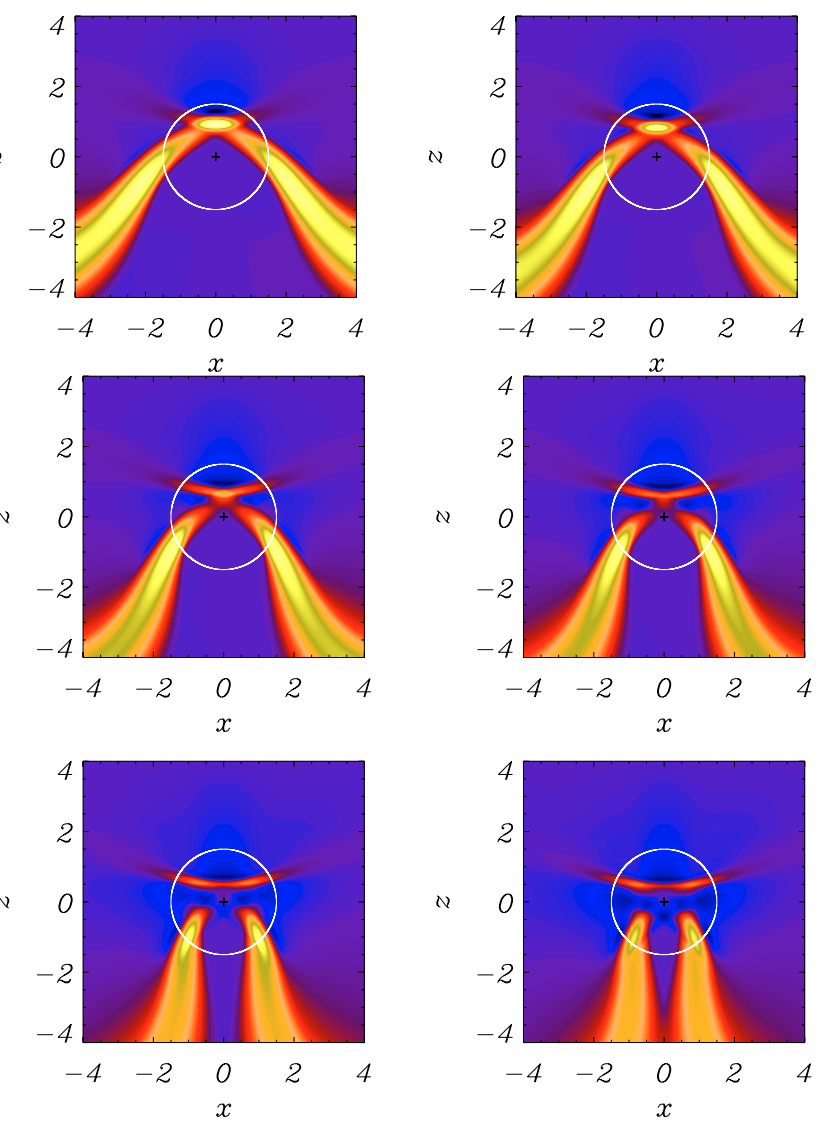

In our numerical simulations, a straight wave pulse in the perpendicular component is sent in from the low $\beta$ region at the top boundary. This disturbance is a low $\beta$ fast wave. At some point the wave will cross the $c_{\mathrm{s}}=v_{\mathrm{A}}$ layer and enter the high $\beta$ environment. Because of the above properties, the incoming fast wave is initially perpendicular to $\boldsymbol{B}_{0}$ but, on crossing the $c_{\mathrm{S}}=v_{\mathrm{A}}$ layer, part of this perpendicular component remains as a fast wave and part is converted into the slow wave. The first point of contact occurs along $x=0$ and at later times for increasing values of $x^{2}$. Thus, we have a low $\beta$ wave approaching the layer, coupling and mixing inside the layer and emerging as a mixture of high $\beta$ fast and slow waves. Later on, the fast wave leaves the high $\beta$ region and re-enters the low $\beta$ region. Mode conversion can occur at this crossing as well.

\section{MHD wave propagation with $\beta_{0}=2.25$}

In this section, the linearised Eqs. (6) are again solved numerically but for $\beta_{0}=2.25$ so that the $c_{\mathrm{s}}=v_{\mathrm{A}}$ layer is now at a larger radius than above. All other boundary conditions remain the same. The behaviour of the perpendicular component of the magnetoacoustic wave when it is inside the $c_{\mathrm{s}}=v_{\mathrm{A}}$ layer can be seen in Fig. 7. Again, we find that the linear, magnetoacoustic wave travels towards the neighbourhood of the null point and begins to wrap around it (as it did for $\beta_{0}=0.25$ ). In this case, since the $\beta \approx 1$ is now reached earlier, the wave has had less time to refract. Using our terminology and interpretation from Sects. 4 and 5, we identify this as the low $\beta$ fast wave. However, when the wave passes through the $c_{\mathrm{s}}=v_{\mathrm{A}}$ layer, a secondary wave becomes apparent; we identify this as the high $\beta$ slow wave. Thus, the low $\beta$ fast wave has transformed into two high $\beta$ waves (the split can be followed closely in Fig. 7).
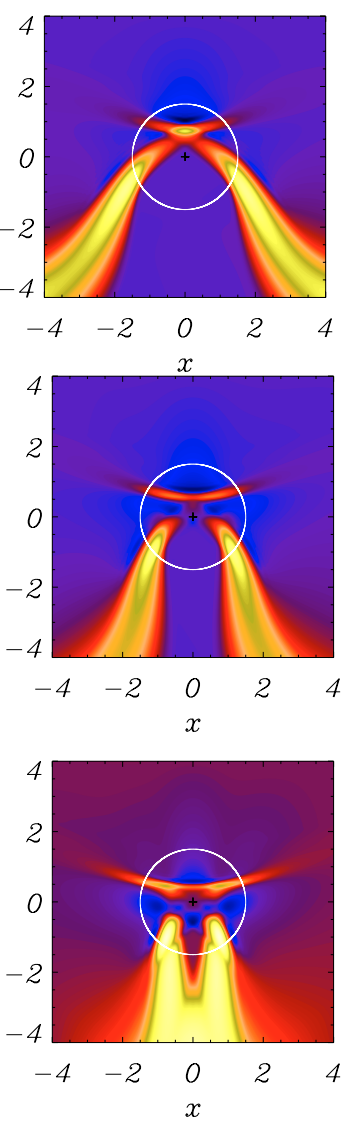

Fig. 7. Contours of $v_{\perp}$ for numerical simulation for a fast wave sent in from upper boundary for $-4 \leq x \leq 4$ and $\beta_{0}=2.25$ and its resultant propagation at times a) $t=1.7$; b) $t=1.8$; c) $t=1.9$; d) $t=2.0$; e) $t=2.1$; f) $t=2.2$; g) $t=2.3$; h) $t=2.4$ and i) $t=2.5$, labelling from top left to bottom right. The white circle indicates the position of the $c_{\mathrm{s}}=v_{\mathrm{A}}$ layer and the cross denotes the null point in the magnetic configuration.

The propagation now proceeds in three ways:

- The generated high $\beta$ slow wave spreads out along the field lines and accumulates along the separatrices. This slow wave cannot cross the separatrices.

- The high $\beta$ fast wave continues to refract and some of the fast wave (located very close to the null point) passes through the null. This effect was not seen in the $\beta=0$ model. The fast wave can now pass through the null because there is now a non-zero fast mode speed, $c_{\text {fast }}$, at the origin. Thus, the high $\beta$ fast wave has passed (slowly) through the null. This crossing still creates a large accumulation of current near the null. However, unlike the $\beta=0$ case, it remains finite. The high $\beta$ fast wave continues to propagate downwards and leaves the $c_{\mathrm{s}}=v_{\mathrm{A}}$ layer, converting to a low $\beta$ fast wave and spreading out (low $\beta$ fast wave propagates roughly isotropically).

- Finally, the rest of the fast wave located away from the null (low $\beta$ fast wave) is not greatly affected by the non-zero sound speed (as $v_{\mathrm{A}}^{2} \gg c_{\text {sound }}^{2}$ ) and so continues to refract around the null. In fact, as the wings of the low $\beta$ fast wave wrap around below the null point, they encounter the part of the fast wave that has travelled through the null and is leaving the $c_{\mathrm{s}}=v_{\mathrm{A}}$ layer. This results in a complicated interference pattern. Nonetheless, it appears that the two fast waves passes through each other without influencing each other (due to the linear nature of the system). A full non-linear treatment of the equations may reveal a different behaviour.

Thus, we have a different behaviour than that seen for the $\beta_{0}=$ 0.25 case: With a large $\beta_{0}$, the perpendicular component of the velocity has passed through the null (interpreted as a high $\beta$ fast wave crossing the null), whereas with a small $\beta_{0}$, we did not 


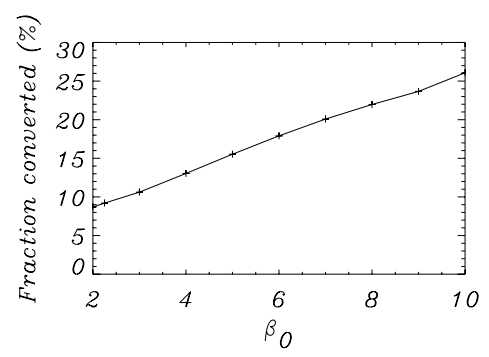

Fig. 8. Fraction of original disturbance converted to high $\beta$ slow wave against parameter $\beta_{0}$, (gradient of straight line is approximately 2.0). Note that for $\beta_{0}<2.0$, the two wave types do not separate enough for us to measure them individually.

see any significant wave pass through the null by the end of the simulation.

So what is the relationship between the $\beta_{0}=2.25$ and $\beta_{0}=$ 0.25 cases; are they not just scaled versions of each other? This will be discussed in the conclusions.

\subsection{Quantifying mode conversion}

At present, there does not exist a robust set of rules connecting low and high $\beta$ waves across the $c_{\mathrm{s}}=v_{\mathrm{A}}$ layer or across the $\beta=1$ layer (Carlsson \& Stein 1999). Here we specifically investigate what happens when a low $\beta$ fast wave crosses the $c_{\mathrm{s}}=v_{\mathrm{A}}$ layer and becomes part high $\beta$ fast wave and part high $\beta$ slow wave (or alternatively what happens when it crosses the $\beta \approx 1$ layer a little time earlier). An obvious question to ask is how much of the incident wave is converted to high $\beta$ slow wave. This is difficult to quantify in our $\beta_{0}=0.25$ simulation, as the high $\beta$ fast and slow waves never really separate enough for us to measure them individually. However, the waves do completely separate for the $\beta_{0}=2.25$ simulation, for example in Fig. $7 \mathrm{~g}$ the slow wave is clearly separated from the other wave types (i.e. the high and low $\beta$ fast waves). Thus, we can measure the amount of this slow wave part by integrating $v_{\perp}$ over the area of the slow wave (to get the volume or, since the equilibrium density is uniform, the total momentum of the slow wave) and compare it to the original incident wave pulse (given by the boundary conditions; Eqs. (9)). This shows that $9.2 \%$ of the initial low $\beta$ fast wave disturbance is converted to high $\beta$ slow wave (for the given boundary conditions with $\beta_{0}=2.25$ ). We can also work out how the proportion of initial disturbance converted to high $\beta$ slow wave changes with $\beta_{0}$. This is shown in Fig. 8 where we can see that the fraction of original disturbance converted to high $\beta$ slow wave is proportional to $\beta_{0}$.

\section{Conclusions}

This paper extends the investigations of McLaughlin \& Hood (2004) into the nature of magnetoacoustic waves in the neighbourhood of a null point. We have conducted two experiments, varying the choice of $\beta_{0}$ in each, where the choice dictated the location of the $c_{\mathrm{s}}=v_{\mathrm{A}}$ layer.

In the first experiment (Sect. 3 ), we set $\beta_{0}=0.25$. We find that the linear magnetoacoustic wave travels towards the neighbourhood of the null point and begins to wrap around it. This occurs due to the spatially varying Alfvén speed, $v_{\mathrm{A}}^{2}(x, z)=x^{2}+z^{2}$, and has been observed before (Nakariakov \& Roberts 1995; McLaughlin \& Hood 2004). In this experiment, we drive a wave pulse in the perpendicular velocity component and we identify this pulse as a low $\beta$ fast wave. However, once the wave reaches and crosses the $c_{\mathrm{s}}=v_{\mathrm{A}}$ layer, part of the wave is transformed into a high $\beta$ slow wave and the majority into the high $\beta$ fast wave. The slow wave part spreads out along the fieldlines. Meanwhile, the rest of the (low $\beta$ ) fast wave continues to wrap around the origin. This refraction effect continues again and again, but each time part of the fast wave is converted to a slow wave as it crosses the $c_{\mathrm{s}}=v_{\mathrm{A}}$ layer. The majority of the current build-up occurs very close to the null.

In our second set of simulations (Sect. 6), we set $\beta_{0}=2.25$, thusplacing the $c_{\mathrm{s}}=v_{\mathrm{A}}$ layer at a larger radius than before (since in this magnetic geometry, the $c_{\mathrm{s}}=v_{\mathrm{A}}$ layer occurs at a radius $\left.r=\sqrt{\frac{\gamma \beta_{0}}{2}}\right)$. In this experiment, we find that the magnetoacoustic wave travels towards the neighbourhood of the null point and begins to wrap around it (as before). However, when the wave crosses the $c_{\mathrm{s}}=v_{\mathrm{A}}$ layer, a secondary wave (high $\beta$ slow wave) becomes apparent (we find that the fraction of incident wave converted to slow wave is proportional to $\beta$ ). The propagation now proceeds in three ways. Firstly, the generated slow wave spreads out along the fieldlines and accumulates along the separatrices. Secondly, the remaining part of the fast wave inside the $c_{\mathrm{S}}=v_{\mathrm{A}}$ layer continues to refract and some of it (located close to the null point) passes through the origin. We identify this part as a high $\beta$ fast wave. The high $\beta$ fast wave can pass through the origin because, although $v_{\mathrm{A}}(0,0)=0$, there is now a non-zero sound speed there (and $c_{\text {fast }}^{2} \approx v_{\mathrm{A}}^{2}+c_{\text {sound }}^{2}$ ). This passing causes a large current accumulation near the origin. After it has crossed the null, the high $\beta$ fast wave continues downwards and leaves the $c_{\mathrm{s}}=v_{\mathrm{A}}$ layer. As it emerges, it becomes a low $\beta$ fast wave and spreads out (since the low $\beta$ fast wave propagates almost isotropically). Finally, the fast wave located away from the null and $c_{\mathrm{s}}=v_{\mathrm{A}}$ layer (the "wings" of the low $\beta$ wave) are not affected by the non-zero sound speed (as $v_{\mathrm{A}}^{2} \gg c_{\text {sound }}^{2}$ ) and so for them the refraction effect dominates. In fact, as these wings wrap around below the null point, they encounter the high $\beta$ fast wave as it is emerging from the $c_{\mathrm{s}}=v_{\mathrm{A}}$ layer. This results in a complicated interference pattern, but it appears that the two waves passes through each other without any lasting effect on each other (due to the linear nature of the system). The $\beta_{0}=2.25$ numerical simulations also showed good agreement with a WKB approximation, until the breakdown point when the neighbouring rays cross each other.

The part of the fast wave that goes through the null effectively escapes the refraction effect of the null point. It seems as if the smaller the value of $\beta_{0}$ used, the slower the high $\beta$ fast wave can cross the null. This can be understood since the high $\beta$ fast wave travels close to the sound speed, which is related to $\beta_{0}$ (recall from Sect. 2.4 that $c_{\mathrm{s}}^{*}=\sqrt{\frac{\gamma}{2} \beta_{0}}$ ). Thus, the behaviour of the $\beta_{0}=2.25$ and $\beta_{0}=0.25$ cases can be understood; in the $\beta_{0}=0.25$ case, the high $\beta$ fast wave can pass through the null, but it travels at such a slow speed (as $c_{\mathrm{s}}$ varies as $\sqrt{\beta_{0}}$ ) that the refraction effect in the low $\beta$ region dominates. Hence, as the $c_{\mathrm{s}}=v_{\mathrm{A}}$ layer gets closer to the null, less and less of the fast wave can pass through the null in a given time, and the parts that can travel through do so at a slower and slower speed. Also, these parts of the wave that do pass through tend to be be swamped by the wings of the rest of the wave wrapping round below the null (repeatedly).

This explains the relationship between our $\beta_{0}=0.25$ and 2.25 investigations. These experiments show scaled versions of each other, but with the larger value of $\beta_{0}$ it is clearer to see that there are actually two competing phenomena; a refraction 
effect caused by the varying Alfvén speed and a non-zero sound speed at the null which allows the fast wave to pass through. It is the value of $\beta_{0}$ that dictates which effect dominates.

Thus, two extremes occur. The first occurs when $\beta_{0} \rightarrow 0$; in which case the refraction effect infinitely dominates over the other effects (and we recover the results of McLaughlin \& Hood 2004) and the second when $\beta_{0} \rightarrow \infty$ and the system becomes hydrodynamic. In this case, the fast wave reduces to an acoustic wave and so completely passes through the null (in effect it does not even see the magnetic field, since $v_{\mathrm{A}}^{2} \ll c_{\mathrm{s}}^{2}$ ). Thus, we can understand the whole spectrum of values of the parameter $\beta_{0}$.

From this work, it has been seen that a warm plasma introduces many new effects not seen in the cold plasma limit, most notably the introduction of fast and slow waves to the system. It also appears that the choice of $\beta_{0}$ is of critical importance, since the two experiments yield different results. However, the key choice here is not in picking $\beta_{0}$, since this just determines where the $c_{\mathrm{s}}=v_{\mathrm{A}}$ layer will occur. The choice of $\beta_{0}$ is equivalent to choosing where to set the boundaries of our box, and so the critical parameter in our system is in choosing the distance between the initial disturbance and the $c_{\mathrm{s}}=v_{\mathrm{A}}$ layer. This is because these experiments do not simply show scaled versions of each other, they show two competing phenomena; a refraction effect caused by the varying Alfvén speed, and a non-zero sound speed at the null which allows the fast wave to pass through.

If the $c_{\mathrm{s}}=v_{\mathrm{A}}$ layer is close to the null (small choice of $\beta_{0}$ and hence there is not much coupling to the parallel velocity and pressure terms) then the refraction effect will dominate, resulting in a scenario similar to $\beta_{0}=0.25$, the waves remain trapped near the null point and heating will occur close to the null. The extreme case is $\beta_{0} \rightarrow 0$, in which case the refraction effect infinitely dominates over the other effects and we recover the results of McLaughlin \& Hood (2004).

However, if the $c_{\mathrm{s}}=v_{\mathrm{A}}$ layer is far from the null, a portion of the fast wave will be able to pass through the origin and escape the system (i.e. will not deposit its energy near the null point). In this case, the remaining wave energy of the generated slow waves is dissipated along the separatrices. So heating will occur in both systems, although the nature will be different.

This is all very interesting for its mathematical sake, but how does the competition of these two effects manifest itself in the corona? The plasma $\beta$ parameter is defined as the ratio of the thermal plasma pressure to the magnetic pressure. In most parts of the corona, $\beta \ll 1$ and hence the pressure gradients in the plasma can be neglected. Values of $\beta=0.01$ are often quoted (e.g. Priest 1982). However, near null points the plasma $\beta$ can become very large, so the (true) plasma $\beta$ varies through the whole region. However as seen above, it is the distance between the initial (planar) pulse and the $c_{\mathrm{S}}=v_{\mathrm{A}}$ layer that is of critical importance. We believe that coronal disturbances will propagate for some distance before they encounter a (coronal) null point. Hence, the fast wave part of the disturbance will feel the refraction effect of the null and begin to refract around it. By the time the $c_{\mathrm{s}}=v_{\mathrm{A}}$ layer is reached or the sound speed becomes important, the fast wave disturbance will be (almost) circular in nature and will have thinned and dissipation will be extracting the energy from the wave. It is true that some of the wave may pass through the null or be converted into high $\beta$ slow waves, but it is likely that the majority of the wave energy will accumulate close to the null, causing large current accumulation and heating there.

Acknowledgements. James McLaughlin acknowledges financial assistance from the Particle Physics and Astronomy Research Council (PPARC). He also wishes to thank Tom Bogdan, Toni Díaz and Erwin Verwichte for helpful and insightful discussions.

\section{References}

Berghmans, D., \& Clette, F. 1999, Sol. Phys., 186, 207 Beveridge, C., Priest, E. R., \& Brown, D. S. 2002, Sol. Phys., 209, 333 Bogdan, T. J., Hansteen, M. C. V., McMurry, A., et al. 2003, ApJ, 599, 626 Brown, D. S., \& Priest, E. R. 2001, A\&A, 367, 339 Bulanov, S. V., \& Syrovatskii, S. I. 1980, Fiz. Plazmy, 6, 1205 Cally, P. S., \& Bogdan, T. J. 1997, ApJ, 486, L67

Cally, P. S. 2001, ApJ, 548, 473

Carlsson, M., \& Stein, R. S. 1999, ASP Conf. Ser., 184, 206

Craig, I. J. D., \& McClymont, A. N. 1991, ApJ, 371, L41

Craig, I. J. D., \& Watson, P. G. 1992, ApJ, 393, 385

Craig, I. J. D., \& McClymont, A. N. 1993, ApJ, 405, 207

De Moortel, I., Hood, A. W., Ireland, J., \& Arber, T. D. 1999, A\&A, 346, 641

De Moortel, I., Ireland, J., \& Walsh, R. W. 2000, A\&A, 355, L23

De Moortel, I., Hood, A. W., Gerrard, C. L., \& Brooks, S. J. 2004, A\&A, 425, 741

Edwin, P. M., \& Roberts, B. 1983, Sol. Phys., 88, 179

Ferraro, C. A., \& Plumpton, C. 1958, ApJ, 127, 459

Heyvaerts, J., \& Priest, E. R. 1983, A\&A, 117, 220

Hood, A. W., Brooks, S. J., \& Wright, A. N. 2002, Proc. Roy. Soc., A458, 2307

McLaughlin, J. A., \& Hood, A. W. 2004, A\&A, 420, 1129

McLaughlin, J. A., \& Hood, A. W. 2006, A\&A, 452, 603

Parker, E. N. 1991, ApJ, 376, 355

Priest, E. R. 1982, Solar Magnetohydrodynamics, D. Reidel Publishing Company

Roberts, B. 1985, Chapter 3: Solar System Magnetic Fields (ed. E. R. Priest), Reidel

Rosenthal, C. S., Bogdan, T. J., Carlsson, M., et al. 2002, ApJ, 564, 508

Nakariakov, V. M., \& Roberts, B. 1995, Sol. Phys., 159, 399

Nakariakov, V. M., Roberts, B., \& Murawski, K. 1997, Sol. Phys., 175, 93

Nakariakov, V. M., Ofman, L., Deluca, E. E., Roberts, B., \& Davila, J. M. 1999, Science, 285, 862

Zhugzhda, I. D., \& Dzhalilov, N. S. 1982, A\&A, 112, 16 


\section{Online Material}




\section{Appendix A: lobe generation}

We can gain insight into the $\beta \neq 0$ system using a small $\beta_{0}$ expansion of the linearised ideal MHD equations. In polar coordinates, our magnetic field is

$\boldsymbol{B}_{0}=-r \cos 2 \theta \hat{\boldsymbol{r}}+r \sin 2 \theta \hat{\boldsymbol{\theta}}$

We now assume $v_{\perp}$ and $v_{\|}$can be expanded in powers of $\beta_{0}$ such that:

$v_{\perp}=v_{\perp 1}(r, t)+\beta_{0}^{2} v_{\perp 2}(r, \theta, t), \quad v_{\|}=\beta_{0} v_{\| 1}(r, \theta, t)$.

Substituting these forms into Eqs. (6) gives:

$$
\begin{aligned}
\frac{\partial^{2}}{\partial t^{2}} v_{\perp 1} & =r \frac{\partial}{\partial r}\left(r \frac{\partial}{\partial r} v_{\perp 1}\right)+O\left(\beta_{0}\right) \\
\frac{\partial}{\partial t} v_{\| 1} & =-\frac{1}{2}\left(\boldsymbol{B}_{0} \cdot \nabla\right) p_{1}+O\left(\beta_{0}\right) \\
\frac{\partial}{\partial t} p_{1} & =-\gamma \nabla \cdot\left[\left(\frac{\sin 2 \theta}{r} v_{\perp 1}\right) \hat{\boldsymbol{r}}+\left(\frac{\cos 2 \theta}{r} v_{\perp 1}\right) \hat{\boldsymbol{\theta}}\right]+O\left(\beta_{0}\right) \\
& =\gamma \sin 2 \theta r \frac{\partial}{\partial r}\left(\frac{v_{\perp 1}}{r^{2}}\right)+O\left(\beta_{0}\right) .
\end{aligned}
$$

Substituting $\frac{\partial}{\partial t} p_{1}$ into the equation for $\frac{\partial}{\partial t} v_{\|_{1}}$ and ignoring terms of order $\beta_{0}$ gives:

$$
\begin{aligned}
\frac{\partial^{2}}{\partial t^{2}} v_{\|_{1}}= & \frac{\gamma}{2}\left\{-r \cos 2 \theta \sin 2 \theta \frac{\partial}{\partial r} r\left[\frac{\partial}{\partial r}\left(\frac{v_{\perp 1}}{r^{2}}\right)\right]\right. \\
& \left.+r \sin 2 \theta \cos 2 \theta\left(\frac{v_{\perp 1}}{r^{2}}\right)\right\} \\
= & \frac{\gamma}{4} \sin 4 \theta r^{3} \frac{\partial}{\partial r} \frac{1}{r}\left[\frac{\partial}{\partial r}\left(\frac{v_{\perp 1}}{r^{2}}\right)\right]=\frac{\gamma}{4} \sin 4 \theta \mathcal{F}(r, t)
\end{aligned}
$$

where $\mathcal{F}(r, t)$ is a known function that depends only on $r$ and $t$. This function is determined solely by the behaviour of $v_{\perp 1}$. Although the shape of the $v_{\perp 1}$ pulse may be continuous, the derivatives in (12) mean that $v_{\|}$may have discontinuous leading and trailing edges. Thus, we can see that, with our choice of magnetic null point and by driving $v_{\perp}$, the form of $v_{\|}$naturally develops a $\sin 4 \theta$ dependence. Assume an initial circular pulse

$v_{\perp 1}=\left\{\begin{array}{cc}\sin \pi(r-2.5) & 2.5<r<3.5 \\ 0 & \text { elsewhere }\end{array}\right.$

The right hand side of (12) is $\frac{\gamma}{4} \sin 4 \theta \frac{\sqrt{3}}{r^{2}}\left[\left(8-\pi^{2} r^{2}\right)\right.$ $\sin \pi(r-2.5)-5 \pi r \cos \pi(r-2.5)]$ and a contour plot of this is shown in Fig. A.1. However, the contour shown is not a true integral of such an initial condition (i.e. is not a solution of (12)) but it does clearly demonstrate the $\sin 4 \theta$ dependence. From Eq. (11), we can also see that $p_{1}$ develops a $\sin 2 \theta$ behaviour. This behaviour can be seen on the right-hand side of Fig. A.1, which shows the contours of $p_{1}$ from a numerical simulation.

\section{Appendix B: analytical work}

In order to gain more insight into the numerical simulations, we use a geometrical optics WKB solution to obtain an approximate analytical solution. By combining Eqs. (2)-(4), we can form:

$$
\frac{\partial^{2} \boldsymbol{v}}{\partial t^{2}}=\frac{\gamma p_{0}}{\rho_{0}} \nabla(\nabla \cdot \boldsymbol{v})+\left\{\nabla \times\left[\nabla \times\left(\boldsymbol{v} \times \boldsymbol{B}_{0}\right)\right]\right\} \times \frac{\boldsymbol{B}_{0}}{\mu \rho_{0}}
$$

We substitute $\boldsymbol{v}=\boldsymbol{a} \mathrm{e}^{\mathrm{i} \phi(x, z)} \cdot \mathrm{e}^{-\mathrm{i} \omega t}$ into (13) where $\boldsymbol{a}$ is a constant amplitude vector in the geometrical optics approximation. The physical optics approximations would allow the amplitude vectors to vary with position as well as the wavenumber $\phi$. Thus, we follow the ray paths. We now take the dot product with $\boldsymbol{B}_{0}$ and $\nabla \phi$ to get the two velocity components:

$$
\left[\begin{array}{cc}
\omega^{2} & -\frac{\gamma p_{0}}{\rho_{0}}\left(\boldsymbol{B}_{0} \cdot \nabla \phi\right) \\
\frac{1}{\mu \rho_{0}}\left(\boldsymbol{B}_{0} \cdot \nabla \phi\right)|\nabla \phi|^{2} & \omega^{2}-\left(\frac{\gamma p_{0}}{\rho_{0}}+\frac{\left|\boldsymbol{B}_{0}\right|^{2}}{\mu \rho_{0}}\right)|\nabla \phi|^{2}
\end{array}\right]\left(\begin{array}{l}
\boldsymbol{v} \cdot \boldsymbol{B}_{0} \\
\boldsymbol{v} \cdot \nabla \phi
\end{array}\right)=\left(\begin{array}{l}
0 \\
0
\end{array}\right) .
$$

These two coupled equations must have zero determinant to prevent a trivial solution, and so by evaluating the determinant, substituting $c_{\mathrm{s}}^{2}=\frac{\gamma p_{0}}{\rho_{0}}$ and $v_{0}^{2}=\frac{B^{2}}{\mu \rho_{0}}$, and making the WKB approximation such that $\omega \sim \phi \gg 1$ leads to a first order equation of the form:

$$
\begin{aligned}
\omega^{4}-\omega^{2}\left[c_{\mathrm{s}}^{2}+v_{0}^{2}\left(x^{2}+\right.\right. & \left.\left.z^{2}\right)\right]\left(p^{2}+q^{2}\right) \\
& +c_{\mathrm{s}}^{2} v_{0}^{2}\left(p^{2}+q^{2}\right)(x p-z q)^{2}=0 \\
\Rightarrow \mathcal{F}(x, z, \phi, p, q)= & 0 \\
= & \frac{1}{2}\left[\omega^{4}-\omega^{2}\left[c_{\mathrm{s}}^{2}+v_{0}^{2}\left(x^{2}+z^{2}\right)\right]\left(p^{2}+q^{2}\right)\right. \\
& \left.+c_{\mathrm{s}}^{2} v_{0}^{2}\left(p^{2}+q^{2}\right)(x p-z q)^{2}\right]
\end{aligned}
$$

where $p=\frac{\partial \phi}{\partial x}$ and $q=\frac{\partial \phi}{\partial z}$ and $\mathcal{F}$ is a non-linear PDE. Also note that the Alfvén speed $v_{\mathrm{A}}^{2}=v_{0}^{2}\left(x^{2}+z^{2}\right)$. This PDE can also be written as

$$
\frac{2 \omega^{2}=\left[c_{\mathrm{s}}^{2}+v_{0}^{2}\left(x^{2}+z^{2}\right)\right]\left(p^{2}+q^{2}\right)}{ \pm \sqrt{\left(p^{2}+q^{2}\right)^{2}\left[c_{\mathrm{s}}^{2}+v_{0}^{2}\left(x^{2}+z^{2}\right)\right]^{2}-4 c_{\mathrm{s}}^{2} v_{0}^{2}\left(p^{2}+q^{2}\right)(x p-z q)^{2}} .}
$$

This equation is reminiscent of the dispersion relation for magnetoacoustic waves (e.g. Roberts 1985). This equation contains information about the two wave types. The method to solve this equation is to assume it to be of the form $\left(\omega^{2}-\omega_{\text {slow }}^{2}\right)\left(\omega^{2}-\omega_{\text {fast }}^{2}\right)=$ 0 . Considering the fast wave (so $\omega^{2} \neq \omega_{\text {slow }}^{2}$ ), we can apply the method of characteristics to generate the equations:

$$
\begin{aligned}
& \frac{\mathrm{d} \phi}{\mathrm{d} s}=2 \omega^{2} \\
& \frac{\mathrm{d} p}{\mathrm{~d} s}=-A x-\left(C A^{2} x-2 p A B c_{\mathrm{s}}^{2} v_{0}^{2}\right) / D \\
& \frac{\mathrm{d} q}{\mathrm{~d} s}=-A z-\left(C A^{2} z+2 q A B c_{\mathrm{s}}^{2} v_{0}^{2}\right) / D \\
& \frac{\mathrm{d} x}{\mathrm{~d} s}=C p+\left(A C^{2} p-2 p B^{2} c_{\mathrm{s}}^{2} v_{0}^{2}-2 c_{\mathrm{s}}^{2} v_{0}^{2} x A B\right) / D \\
& \frac{\mathrm{d} z}{\mathrm{~d} s}=C q+\left(A C^{2} q-2 q B^{2} c_{\mathrm{s}}^{2} v_{0}^{2}+2 c_{\mathrm{s}}^{2} v_{0}^{2} z A B\right) / D
\end{aligned}
$$

where $A=p^{2}+q^{2}, B=x p-z q, C=c_{\mathrm{s}}^{2}+v_{0}^{2}\left(x^{2}+z^{2}\right), D=$ $\sqrt{A^{2} C^{2}-4 A B^{2} c_{\mathrm{s}}^{2} v_{0}^{2}}$ and $\omega$ is the frequency of our wave and $s$ is some parameter along the characteristic. These five ODEs were solved numerically using a fourth-order Runge-Kutta method. Contours of constant $\phi$ can be thought of as defining the positions of the edges of the wave pulse, i.e. with correct choices of $s$, the WKB solution represents the front, middle and back edges of the wave.

We see that the agreement between the numerical simulation and the analytical approximation is quite good (see Fig. A.2). In Fig. A.3, we see the main features of the numerical simulation; 

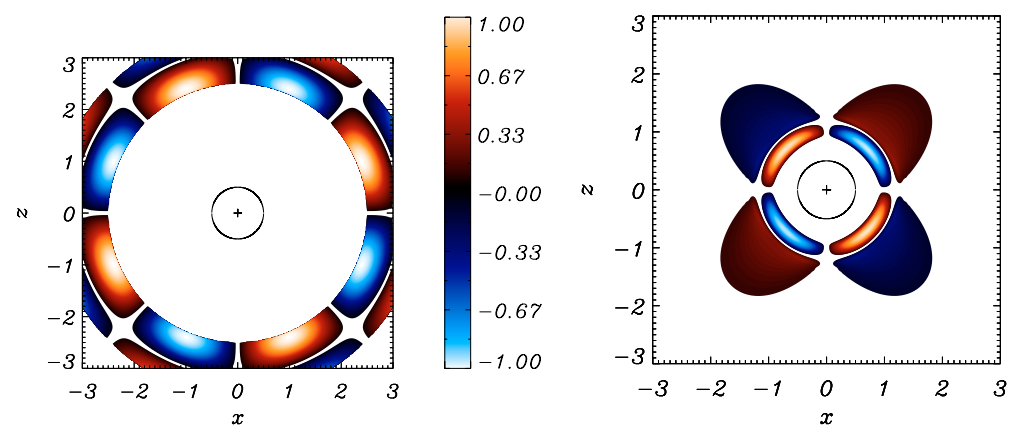

Fig. A.1. (Left) Contour of $\frac{\gamma}{4} \sin 4 \theta \frac{\sqrt{3}}{r^{2}}\left[\left(8-\pi^{2} r^{2}\right) \sin \pi(r-2.5)-5 \pi r \cos \pi(r-2.5)\right]$ for $2.5 \leq r \leq 3.5$. (Right) Contours of the numerical simulation of $p_{1}$ for a fast wave pulse initially located about a radius $\sqrt{x^{2}+z^{2}}=3$ and its resultant propagation after time $t=1.0$. The black circle indicates the position of the $c_{\mathrm{s}}=v_{\mathrm{A}}$ layer and the cross denotes the null point in the magnetic configuration. $p_{1}$ has an alternating form, where orange represents $p_{1}>0$ and blue $p_{1}<0$.
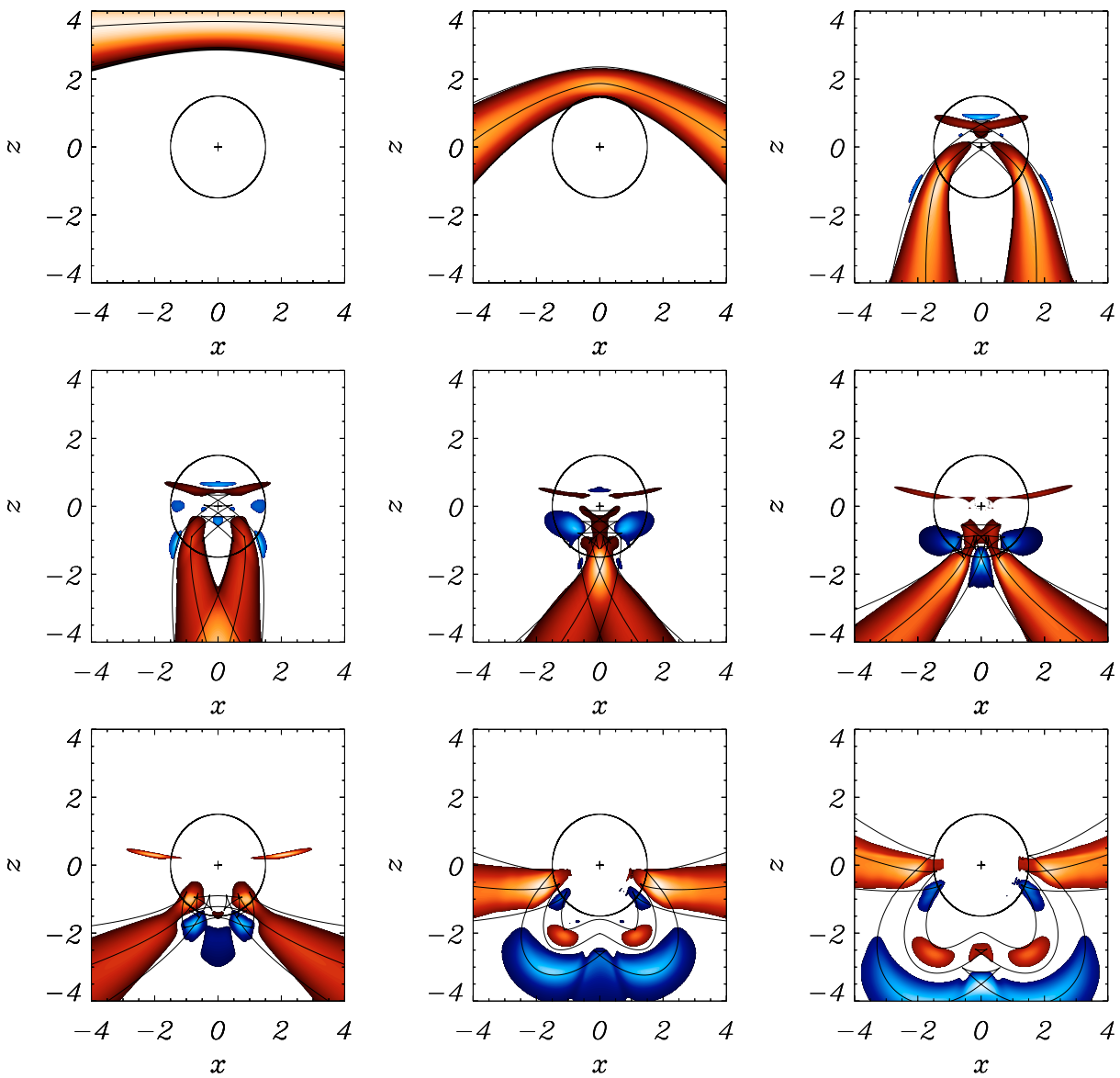

Fig. A.2. Comparison of $v_{\perp}$ from numerical simulation and analytical solution for a fast wave sent in from upper boundary for $-4 \leq x \leq$ 4 and $\beta_{0}=2.25$ and its resultant propagation at times a) $t=0.33$; b) $t=1.0$; c) $t=2.0$; d) $t=2.33$; e) $t=2.67$; f) $t=3.0$; g) $t=$ 3.33 ; h) $t=3.67$ and i) $t=4.0$, labelling from top left to bottom right. The lines represent the front, middle and back edges of the wave. The circle indicates the position of the $c_{\mathrm{s}}=v_{\mathrm{A}}$ layer and the cross denotes the null point in the magnetic configuration.

the wave refracts as it approaches the null and that part of the wave passes through the null (at a slower speed than the "wings" outside the $c_{\mathrm{s}}=v_{\mathrm{A}}$ layer). This central part (that passed through the null) then emerges from the $c_{\mathrm{s}}=v_{\mathrm{A}}$ layer and spreads out isotropically. Meanwhile, the wings continue to refract around the null. Also, as the wave crosses the $c_{\mathrm{S}}=v_{\mathrm{A}}$ layer, the wavefront overlaps with itself (forming small triangular shapes, see Fig. A.4); this may explain why we see the perpendicular wave form a sharp edge as it crosses the $c_{\mathrm{s}}=v_{\mathrm{A}}$ layer. However, the crossing of neighbouring rays frequently indicates the breakdown of the WKB solution, as happens at caustics. This is almost certainly where the conversion of part of the fast wave into a slow wave occurs and this process requires a more detailed study of the WKB equations. Instead of the triangular shape propagating at the fast speed it should propagate at the slow speed.

The rays of the WKB solution can be seen in Fig. A.4. The left graph shows the smalltriangular shape formed as the wavefront overlaps with itself when it crosses the $c_{\mathrm{S}}=v_{\mathrm{A}}$ layer, indicating that this is not just a discontinuity in the wavefront (i.e. since it can be resolved). The central graph shows the rays for starting points of $x=1,2,2.5,3$ and 4 along $z=4$. We see that for a starting point of $x=3$ and $x=4(z=4)$, the ray is deflected substantially by the null point, whereas the deflection is less severe for 2 and 1. A starting point of $x=2.5$ seems to 
J. A. McLaughlin and A. W. Hood: MHD mode coupling in the neighbourhood of a 2D null point, Online Material p 4
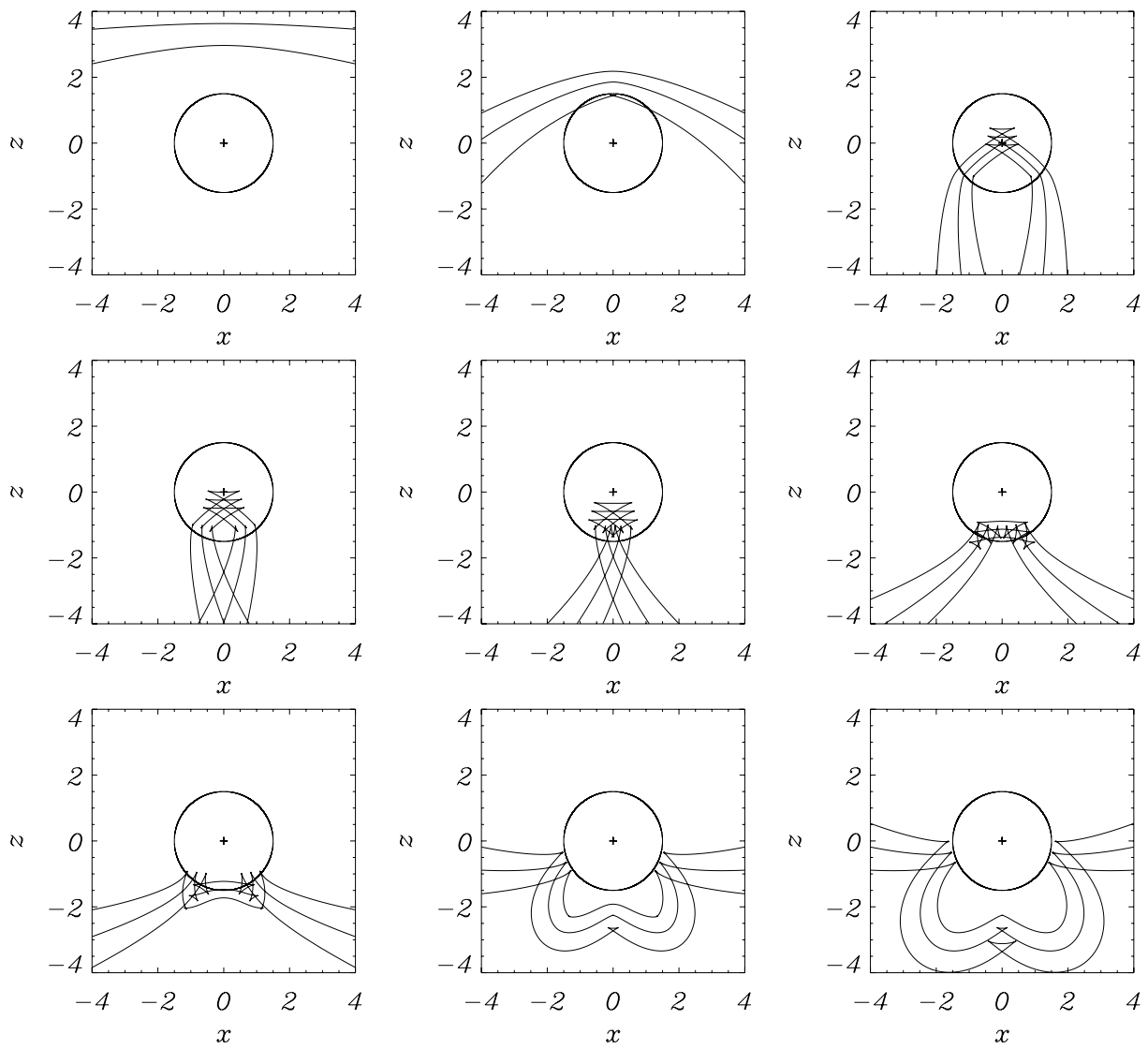

Fig. A.3. Plots of WKB solution for a wave sent in from the upper boundary and its resultant positions at times a) $t=0.33$; b) $t=$ 1.0 ; c) $t=2.0 ; \mathbf{d}) t=2.33$ e) $t=2.67$; f) $t=3.0$; g) $t=$ 3.33 ; h) $t=3.67$ and i) $t=4.0$, labelling from top left to bottom right. The lines represent the front, middle and back edges of the wave. The black circle indicates the position of the $c_{\mathrm{s}}=v_{\mathrm{A}}$ layer and the cross denotes the null point in the magnetic configuration.
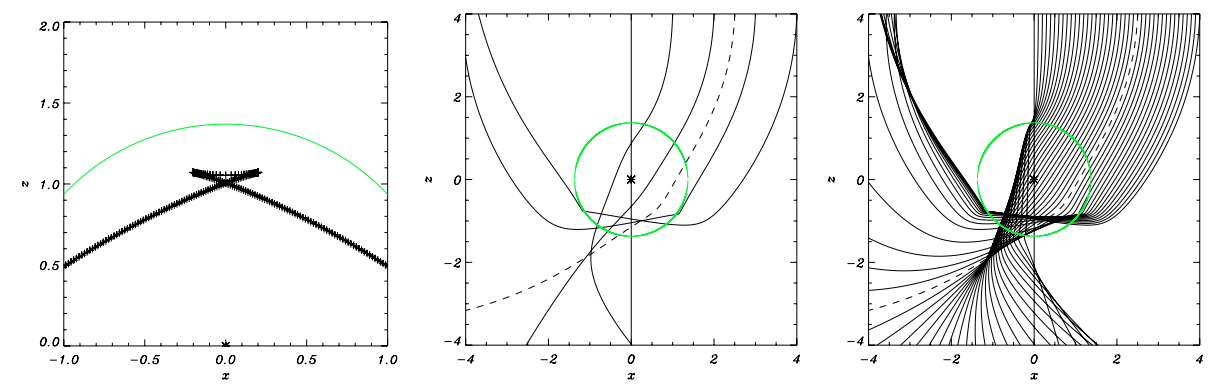

Fig. A.4. Graphs showing various aspects of the analytical WKB approximation. Left shows blow-up of wavefront at time $t=0.65$. Center shows rays for WKB solution for a wave sent in from the upper boundary for starting points of $x=0,1,2,2.5,3$ and 4 along $z=4$. Right shows same rays but for $x \in[0,4]$ at intervals of 0.1 . The green circle indicates the position of the $c_{\mathrm{s}}=v_{\mathrm{A}}$ layer and the star denotes the null point.

be the critical starting point that determines if $\frac{\mathrm{d} z}{\mathrm{~d}}$ changes sign (at least in the range $-4 \leq x \leq 4$ ). We also see that $x=0$ is not deflected at all. The right hand graph shows the rays for for $x \in[0,4]$ in intervals of 0.1 . The dashed line represents $x=2.5$; for $x>2.5$, the ray path is deflected so much that $\frac{\mathrm{d} z}{\mathrm{~d} s}$ changes sign. For $x<2.5$, the rays are deflected but escape from the opposite corner of the system. This nature reflects the numerical solution; part of the wave passes through the null and part refracts around it.
However, the analytical WKB approximation does not give a full description of the numerical simulation. Firstly, the WKB approximation breaks down when neighbouring rays cross and this requires a more detailed study to demonstrate exactly how the fast and slow waves interact. Secondly, the WKB approximation can also be applied to the $\omega^{2}-\omega_{\text {slow }}^{2}=0$ equation but this does not greatly add to our understanding of the system. 